\title{
Aplicação de Ácido Hialurônico em região labial guiado por ultrassonografia de alta frequência com Doppler
}

\section{Hyaluronic acid application in the labial region guided by high-frequency Doppler ultrasound}

DOI: $10.46919 / \operatorname{archv2n2-005~}$

Recebimento dos originais: 01/01/2021

Aceitação para publicação: 31/03/2021

\author{
Adriana Novaes Rodrigues \\ Doutora em Ciências Medicas USP \\ Prof. Coordenadora Curso de Procedimento Médicos Estéticos \\ Instituição/Afiliação Universidade de São Paulo \\ E-mail: a.novaes@live.com \\ Karlos Gudde de Holanda Moura - \\ Especialista em Anestesia \\ Pós graduado em Laser, Cosmiatria e Procedimentos \\ Instituição/Afiliação Faculdade De Tecnologia e Saúde De Ribeirão Preto \\ E-mail: karlos_holanda@hotmail.com
}

João Maluf Franco

Titulação - Pós graduado em Ultrassonografia

Instituição/Afiliação Consultório particular

E-mail: jmaluf@live.com

\section{RESUMO}

O presente artigo aborda, em região labial, a técnica de preenchimento com ácido hialurônico guiado por ultrassom doppler de alta frequência. Objetivo demonstrar que este procedimento, minimiza as complicações vasculares, devido à grande vascularização da região labial. Método: utilização de ultrassom com Doppler e transdutor de alta frequência de $22 \mathrm{MHz}$, em procedimento de preenchimento labial. Resultados: Com a diminuição de riscos e localização exata do produto injetado, propõem-se a utilização do ultrassom guiado para o procedimento de $\mathrm{AH}$ injetável, a fim de minimizar os riscos vasculares, em áreas faciais.

Palavras-chave: Saúde estética, Ácido Hialurônico, Preenchimento labial, Ultrassonografia.

\begin{abstract}
This article addresses, in the labial region, the technique of filling with hyaluronic acid guided by high frequency ultrasound. Objective to demonstrate that this procedure, minimizes vascular complications, due to the great vascularization of the labial region. Method: use of doppler ultrasound, with a $22 \mathrm{MHz}$ probe, in a lip filling procedure. Resultads :With the reduction of risks and exact location of the injected product, it is proposed to use guided ultrasound for the injectable HA procedure, to minimize vascular risks in facial areas.
\end{abstract}

Key words: Aesthetic health. Hyaluronic acid, Lip filling, Doppler Ultrasonography 


\section{INTRODUÇÃO}

A região labial é um componente fundamental para a harmonização facial com diversas abordagens possíveis estéticas. Entre elas a utilização de ácido hialurônico injetável que atualmente é o agente mais utilizado capaz de restabelecer essas características ideais do lábio (Braz, 2009).

Anatomicamente os lábios se apresentam em três partes: uma porção interna, denominada úmida, composta por mucosa labial; por uma porção seca, composta por zona de transição e vermelhão do lábio; e uma porção externa composta de pele e anexos. No limite entre a porção interna e a zona de transição encontram-se as fibras musculares do músculo orbicular da boca que delimita dois compartimentos de gordura, o compartimento de gordura superficial e o compartimento de gordura profundo (Braz; Sakuma, 2017).

Em repouso a anatomia dos lábios é variável, eles podem ser cheios ou finos, quanto ao volume, largos ou estreitos, quanto a largura horizontal, curtos ou largos, quanto a medida subnasal/estômio, visto que a medida vertical do lábio superior em relação ao lábio inferior a proporção ideal na mulher é 1:1,6. (Ballarin, 2018). Já no homem essa proporção é de 1:1.

Embora todas as opções de AH para harmonização labial apresentem boa tolerância, não existe nenhuma técnica de utilização de preenchedores totalmente desprovida de riscos e, além disso, mesmo profissionais experientes podem eventualmente ter intercorrências como complicações precoces arteriais, tendo sido descritos na literatura especializada (Junkins-Hopkins, 2010).

A região labial é extremamente vascularizada, sendo irrigada principalmente pelas artérias labial superior e inferior, que são ramos da artéria facial e, apesar de pouco comuns, as complicações relacionadas ao uso de AH podem ser graves e irreversíveis, como as vasculares. A isquemia labial pode ser causada por embolia arterial de ácido hialurônico ou por compressão extrínseca, com manifestações imediatas, sendo o seu diagnóstico eminentemente clínico. Contudo é a necrose a complicação mais temida.

Utilizado desde os anos 70, o ultrassom tem contribuído na avaliação do espessamento cutâneo (Kleinerman, et al., 2012) Com o avanço da tecnologia e a introdução dos equipamentos de alta frequência, (com ou sem Doppler) houve melhora da resolução, possibilitando a observação de estruturas superficiais, fornecendo uma análise mais detalhada da pele (Bagatin, et al., 2013). Esta análise propiciou a introdução da ultrassonografia como um recurso para minimizar as complicações em decorrência dos procedimentos. É importante salientar que quanto maior a frequência do transdutor maior será a definição na imagem entre dois pontos próximos entre si, o que permite definir na imagem a distinção da epiderme e da derme e suas respectivas camadas. As espessuras das camadas epiderme e derme variam de 0,05 a $0,10 \mathrm{~mm}$ e 1,2 a 1,8 $\mathrm{mm}$, respectivamente. Baseando-se nisso, os autores propõem a utilização do ultrassom guiado com Doppler para o procedimento de preenchimento labial com AH injetável, afim de minimizar os riscos vasculares inerentes a essa técnica. 


\section{MÉTODO}

Precedendo o início da avaliação cutânea no intuito de evitar infecções faz-se necessário a remoção de maquiagens, quando houver, e realização de antissepsia com clorexidina alcoólica 0,5\% seguida de enxágue bucal antes do procedimento injetável, a fim de reduzir a microbiota oral. O enxaguante bucal com clorexidina oral a 0,2\% é o mais eficaz na redução do biofilme dental in vivo (Quincas, et al., 2015).

O mapeamento da vascularização arterial facial, pré procedimento de $\mathrm{AH}$, localiza e evidencia as artérias labial superior e labial inferior que podem ser encontradas na submucosa, intramuscular ou subcutânea, entretanto a localização mais frequente delas é na camada submucosa. ( Fig 1)

Fig 1. Artéria Labial Superior e Inferior

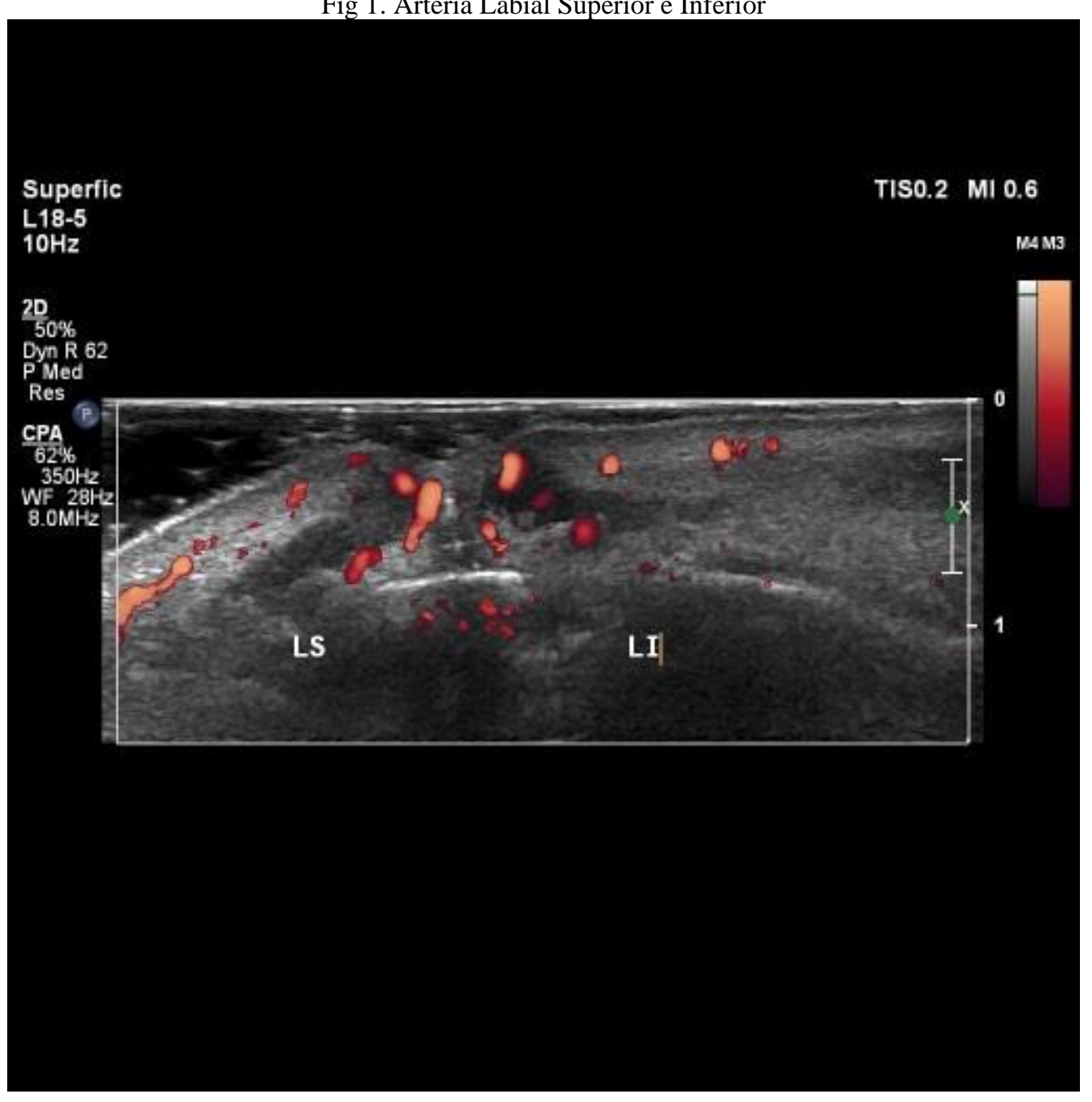

Para o mapeamento foi utilizado ultrassom com transdutor de alta frequência no modo B e Doppler, sendo o transdutor escolhido para este tipo de procedimento o linear de $18 \mathrm{MHz}$ baseado na literatura especializada vigente (Barcaui et al., 2015). 
A diferenciação entre artéria e veia pode ser realizada com a ultrassonografia bidimensional de compressão ou com o uso do Doppler pulsado, seja espectral ou colorido, ainda que não haja superioridade de um sobre o outro (Rupp, et al., 2012).

Na técnica dinâmica a abordagem ecográfica acontece em tempo real e todo o procedimento de injeção do fármaco é guiado pelo Modo B com Doppler (Fig 2), respeitando-se os cuidados de assepsia com o uso de bainha e gel estéreis.

Fig.2: Ultrassonografia labial ALI com Doppler

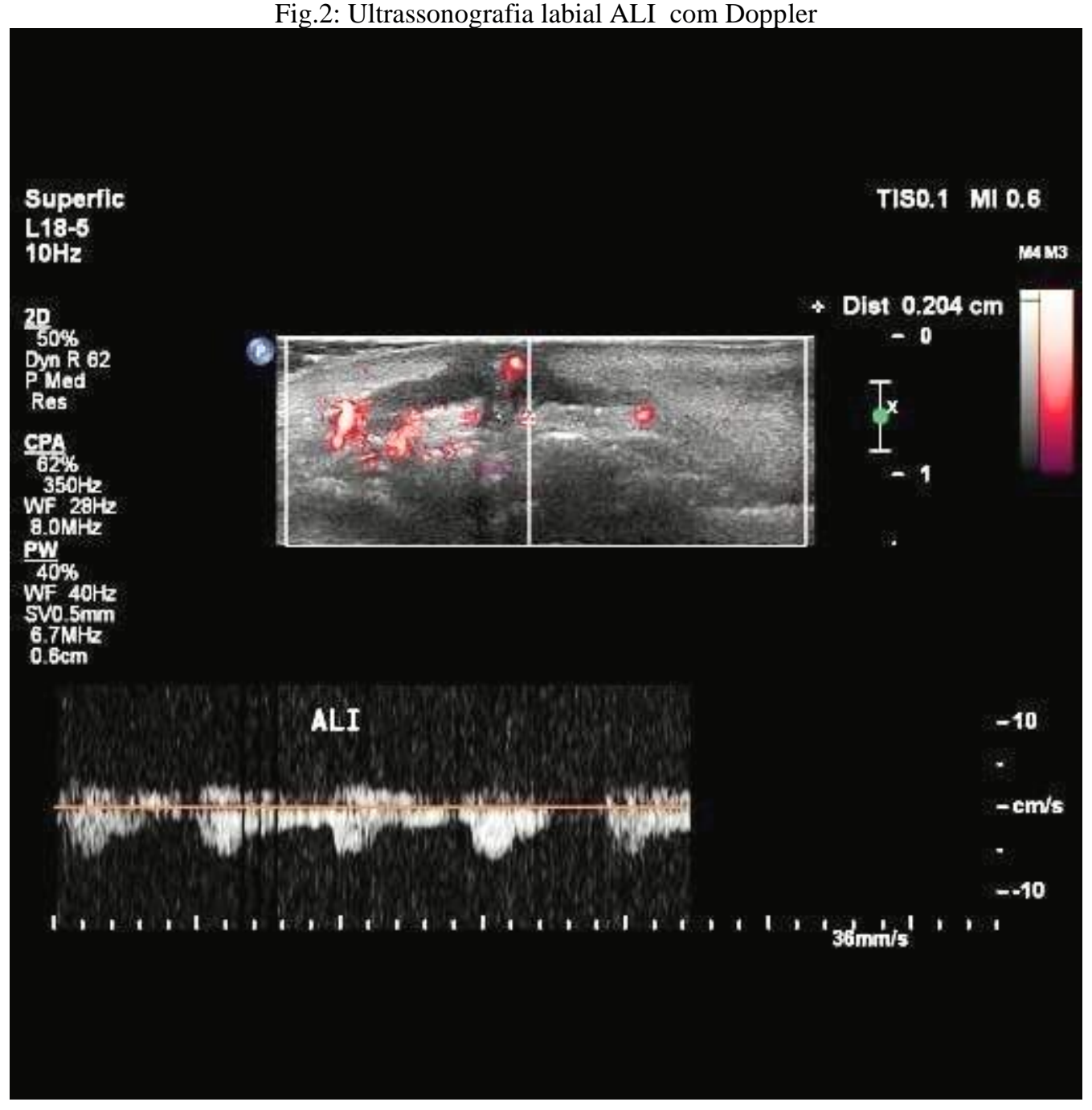

Durante o procedimento, a cânula $25 \mathrm{G}$ é inserida a $1 \mathrm{~cm}$ da comissura labial e direcionada para o lábio superior, na transição da área seca para a molhada do vermelhão labial. Após sua inserção um mapeamento intraprocedimento com a ultrassonografia com Doppler é realizado com o objetivo de visibilizar a vascularização arterial e sua relação com a cânula inserida. Uma vez constatado que não há lesão vascular, inicia-se a retroinjeção do ácido hialurônico para realização do preenchimento. A cânula é redirecionada para o lábio inferior e o mesmo procedimento é realizado. A finalização do procedimento 
ocorre quando a mesma técnica é realizada no lado contralateral. O preenchedor utilizado associa ácido hialurônico reticulado $20 \mathrm{mg} / \mathrm{ml}$ à lidocaína $3 \mathrm{mg} / \mathrm{ml}$, e seu uso é específico para lábios.

A realização da ultrassonografia pós procedimento, fornece dados valiosos para avaliação, quanto a complicações vasculares, do paciente. (Fig 3)

Fig 3: USG após aplicação ácido Hialurônico

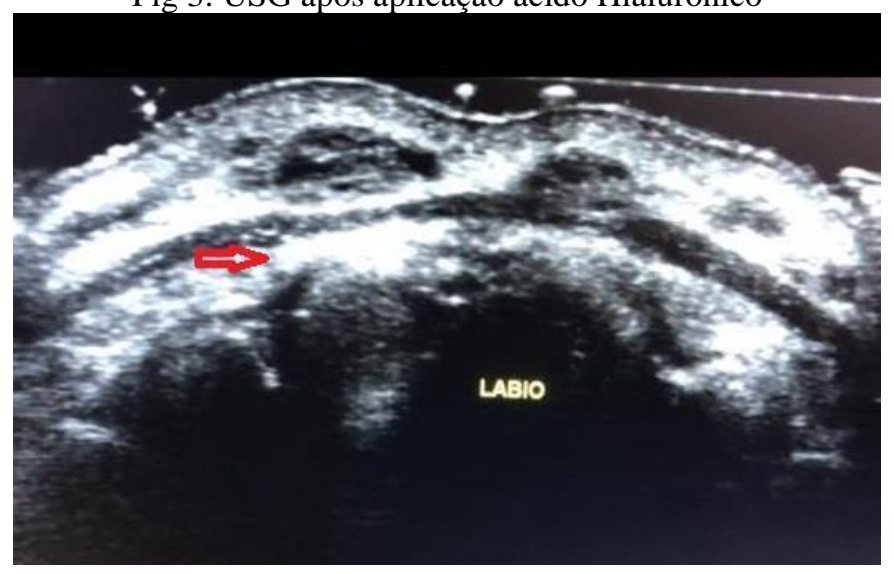

\section{DISCUSSÃO}

A avaliação do paciente previamente ao procedimento de aplicação de AH é fundamental para relacionar o risco envolvido ao procedimento. Uma anamnese bem realizada, verificando desde a expectativa dos pacientes, até a determinação do produto a ser utilizado, minimizam os riscos (Laffaile e Benedetto, 2010). Porém, as variações anatômicas decorrentes da rica vascularização facial pode comprometer o procedimento.

A oclusão vascular é a complicação mais preocupante e resulta da injeção intravascular direta ou a compressão dos vasos pelo preenchedor injetado. São consideradas áreas de alto risco para aplicação de AH a glabela, a região temporal, a região nasal e o sulco nasolabial. Estas áreas estão associadas ao risco de lesão ocular, uma vez que a retroinjeção de AH pode levar à oclusão da artéria central da retina cuja consequência é a amaurose. A oclusão arterial pode ainda resultar desde a necrose local ou segmentar da pele, com ou sem oclusão a distância e até mesmo eventos isquêmicos cerebrais (Abduljabbar e Basendwh, 2016).

No caso de injeção intravascular acidental ao se utilizar preenchedores de AH, deve-se realizar uma investigação da área afetada e de sua vascularização, inciando o mais precoce possível a injeção da Hialuronidase em altas concentrações no intuito de desobstruir a artéria afetada.

Esta é a grande vantagem da utilização da aplicação de AH guiado por ultrassom com Doppler. Com o uso dessa técnica ultrassonográfica, guiando e/ou mapeando a vascularização da face é possível tornar o procedimento muito mais seguro. Mesmo que o médico injetor tenha domínio absoluto da anatomia da 
vascularização facial, ele não está imune às variações anatômicas dos pacientes, não sendo possível verificar a compressão extrínseca dos vasos antes da clínica ser instalada.

A ultrassonografia diagnóstica com Doppler permite a avaliação cuidadosa dos pacientes para os quais a injeção percutânea de AH evita a punção de vasos aberrantes e estima a distância da superfície da pele à área do procedimento, assegurando ao mesmo tempo a colocação precisa da agulha e/ou cânula na região tratada. O uso da ultrassonografia torna-se assim, o melhor meio para minimizar as complicações vasculares. (Koundal, et al., 2019).

\section{CONCLUSÕES}

O mapeamento da vascularização facial guiada por ultrassonografia com Doppler demostrou uma maior segurança durante o procedimento de injeção de $\mathrm{AH}$. A sua superioridade em relação à técnica tradicional, tanto pela eficácia quanto pela segurança, retratados neste artigo, propicia um meio de maior segurança ao profissional médico na sua atividade laboral diária. Importante destacar que esse método se aplica a qualquer área da face onde seja necessária a realização do preenchimento com $\mathrm{AH}$. 


\section{REFERENCIAS}

ABDUlJABBAR, M. H.; BASENDWH, M. A. Complications of hyaluronic acid fillers and their managements - Journal of Dermatology \& Dermatologic Surgery 20 (2016) 100-106.

BAGATIN E, CAETANO LVN, SOARES JLM. Ultrasound and dermatology: basis principles and main applications in dermatologic research. Expert Rev of Dermatol. Oct. 2013;8:463-77.

BALLARIN, A. Escultura Labial B3D: A Harmonização da Face, Sorriso e Lábios. In: CARBONE, A. MDM - Harmonização $\quad$ Orofacial 2018. BARCAUI EO, CARVALHO ACP, PIÑEIRO-MACEIRA J, BARCAUI CB, MORAES H. Estudo da anatomia cutânea com ultrassom de alta frequência $(22 \mathrm{MHz})$ e sua correlação histológica. Radiol Bras. 2015 Set/Out;48(5):324-329.

BRAZ AV. Update no tratamento com ácido hialurônico. In: Kede MPV, Sabatovich O, editores. Dermatologia Estética. São Paulo: Ateneu; 2009. p. 646-61.

BRAZ, A.V.; SAKUMA, T.H. Compartimento de gordura profundo (CGP). In: Atlas de anatomia e preenchimento global da face . Rio de Janeiro: Guanabara Koogan, 2017. p. 29-113.

CHONG BF, BLANK LM, MALAUGJLIN R, NIELSEN LK. Microbial hyaluronic acid production. Appl Microbiol Biotechnol. 2005;66(4):341-51.

DAHER JC, DA-SILVA SV, CAMPOS AC, DIAS RCS, DAMASIO AA, COSTA RSC. Complicações vasculares dos preenchimentos faciais com ácido hialurônico: confecção de protocolo de prevenção e tratamento. Rev.Bras.Cir.Plást.2020;35(1):2-7.

DELORENZI C. Complications of injectable fillers, part I. Aesthet Surg J. 2013;33(4):561-75.

DELORENZI C. New High Dose Pulsed Hyaluronidase Protocol for Hyaluronic Acid Filler Vascular Adverse Events. Aesthet Surg J.2017;37(7):814-25.

HWANG, C. Periorbital injectables: understanding and avoiding complications - J Cutan Aesthet Surg. 2016; 9 (2): 7379.

JUNKINS-HOPKINS JM. Filler complications. J Am Acad Dermatol. 2010;63(4):703-5.

JUNQUEIRA LC \& CARNEIRO J. (2013). Histologia básica. Rio de Janeiro, Brasil .

KLEINERMAN R, WHANG TB, BARD RL, et al. Ultrasound in dermatology: principles and applications. J Am Acad Dermatol. 2012;67:478- 87.

KOUNDAL V., RANA S., THAKUR R., CHAUHAN V., EKKE S., KUMAR M. The usefulness of point of care ultrasound (POCUS) in preanaesthetic airway assessment Indian Journal of Anaesthesia, Volume $63,2019$.

LAFAILLE P, BENEDETTO A. Fillers: contraindications, side effects and precautions. Journal of cutaneous and aesthetic surgery. 2010;3(1):16-9. QUINTAS V, PRADA-LÓPEZ I, DONOS N, SUÁREZQUINTANILLA D, 
RUPP SM, APFELBAUM JL, BLITT C, CAPLAN RA, CONNIS RT, DOMINO KB, et al. Practice Guidelines for Central Venous Access. A Report by the American Society of Anesthesiologists Task Force on Central Venous Access. Anesthesiology. 2012; 116(3): 539-73.

TOMÁS I. Antiplaque effect of essential oils and $0.2 \%$ chlorhexidine on an in situ model of oral biofilm growth: a randomised clinical trial. PLoS One. 2015;10(2).

ZEUGOLIS DI \& RAGHUNATH M. (2011). Collagen: Materials Analysis and Implant Uses. In: P. DUCHEYNE, K. HEALY, D. HUTMACHER, D. GRAINGER, C. KIRKPATRICK (Eds.). Comprehensive 